\title{
The Shape Characteristic Constitution of the Aggregation Based on Pro/Engineer in Engineering Graphics Education Innovation
}

\author{
Hou Zhiyan
}

College of Mechanical Engineering Taiyuan University of Technology Taiyuan, China

\begin{abstract}
The engineering graphics is an old course with comparatively fixed content, but the teaching system should couple with the development of modern society. The new technique should be led into the course in time, so as to make the course develop vigorously. With the wide application of the Pro/Engineer which is a three-dimensional parameter software, introducing it into the course is very necessary. This article introduces the application of Pro/Engineer in the teaching of the Aggregation, puts forward the new idea of the characteristic constitution of the Aggregation, solves inadequate space imagination and enhances the association with the course such as machine design in the aspect of the constitution and expression technology of the machine, and well trains students' creative design ability on the term of earlier period.
\end{abstract}

Index Terms: pro/engineer; characteristi constitution; aggregation; the shape characteristic;

(C) 2012 Published by MECS Publisher. Selection and/or peer review under responsibility of the International Conference on E-Business System and Education Technology

\section{Introduction}

The "engineering design" [1] which many graphics educator advocate is the main idea of engineering graphics reform in recent years. The drawing is based on the design, which is a tool of expression design concept as well as a manifestation of the design achievements. Traditional engineering graphics course content involves few design modeling, so how to coordinate "the drawing" and "design ability" is the key of education reform, so as to improve students' space imaginative ability and the configuration design capacity. Moreover, Pro/Engineer[2] is an extensive and profound three-dimensional parameter software which associates the engineering practice tightly, and covers the whole process of product from conceptual design, industry shape design, three-dimensional model design, analytic calculation, dynamic analog simulation, the out-putting of engineering drawing to production, so the way of "characteristic constitution" used in establishing three-dimensional model brings the new idea about the understanding of constitution of the aggregation. The concept of the characteristic is introduced as fellows. 


\section{THECONCEPT AND CLASSIFICATION OF THE CHARACTERISTIC IN THE PRO/ENGINEER}

The characteristic is the geometry entity which has relatively particular shape, attribute and particular design and manufacturing meaning. For example, the designing of a bore, according to the design system of characteristic, the bore is a characteristic which has attributes such as diameter, length, position, tolerance and roughness...etc. Each characteristic can be processed through a set of the manufacturing method.

The information model of the parts [3] should include: manage characteristic, shape characteristic, accuracy characteristic, material characteristic and technique characteristic. The shape characteristic, which is an essential element that constructs part shape, is the most important characteristic in the part model. It can provide commonly shared product, shape information for design and manufacturing. The management, control and use of shape characteristic is an effective method of converting the information of design to the manufacturing information, therefore, the shape characteristic is thought to be a communication medium during design and manufacturing. Furthermore, based on the geometry, design and manufacturing. The shape characteristic is thought to be particular shape constituted by a group of geometry elements (dot, line, surface) which have certain topology relation. It can not only describe the relation of characteristics, but also correspond to the fixed processing way, so it is often used in the design, analysis and manufacturing.

The shape characteristic of the parts is not only the main stem that constructs parts model, but also the bridge of contact design characteristic and craft characteristic, so it is the basis of designing. While designing a product [4], usually to meet the demands of the function and property of the product, a series of shape characteristics that have certain function construct a product. Consequently, the shape characteristic is thought to be not only the focus of designing, but also the information carrier. A shape characteristic is mainly introduced as follows.

\section{THE CONSTITUTION WAY OF SHAPE CHARACTERISTIC OF THE AGGREGATION}

The Aggregation [5] can be thought as the part that is abstract and oversimplified. How to construct the aggregation closely relates to the course in the follow-up study on the mechanical engineering, such as Mechanical Design and Technology of Mechanical Manufacture...etc. In the old content of courses of the Engineering Graphics, the aggregation is constructed by the basic polyhedral solid and the basic curved solid among which overlay or cut. With the application of the Pro/E in the teaching of aggregation in the Engineering Graphics, the new idea about the constitution way of the aggregation occurred. The Aggregation can be constructed by a series of shape characteristics which strengthens the contact with contents in the part drawing, moreover, lays the substantial foundation on the creativity design ability. Kinds of familiar shape characteristic to construct the aggregation are introduced below.

\section{1 The way to construct the aggregation based on the stretching characteristic.}

The way to construct the aggregation based on the stretching characteristic is to stretch the plane figure a certain distance along its normal line, then the plane figure becomes the aggregation. If all sides of the plane figure are straight lines, then a body gained is to be called flat edge pillars; If the sides of the plane figure include curved line like arc, etc, then a body gained is to be called the curve edge pillar, as shown in the figure 1.

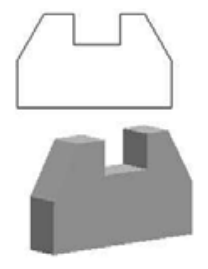

(a)

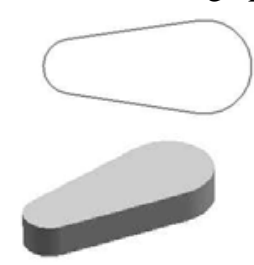

(b)

fig 1 (a) . flat edge pillars（b) . curve edge pillar 
3. 2 The way to construct the aggregation based on the revolving characteristic.

The way to construct the aggregation based on the revolving characteristic is to revolve the plane figure along a certain axis, then the plane figure becomes the aggregation which is called solid of revolution, as is shown in the figure 2 .

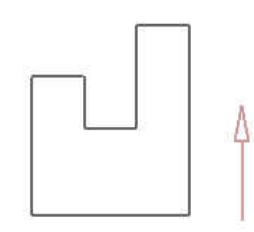

(a)

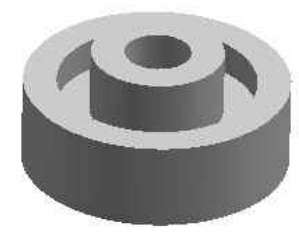

(b)

fig 2. solid of revolution

\section{3 The way to construct the aggregation based on the overlaying characteristic}

The way to construct the aggregation based on the overlaying characteristic is to overlay the uncomplicated pillars as is shown in the figure 3(a). Then the aggregation as is shown in the figure 3(b) forms.

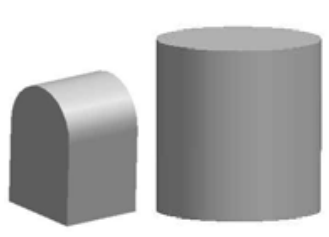

(a)

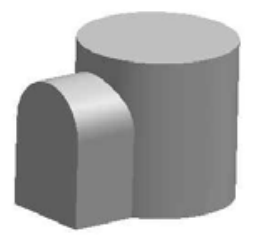

(b)

fig 3. solid of overlaying

\section{4 The way to construct the aggregation based on the cutting-out characteristic}

The way to construct the aggregation based on the cutting-out characteristic is to cut some small simple shape out of a big body, as is shown in the figure 4.

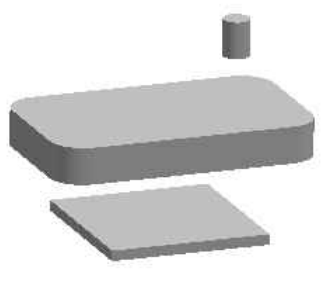

(a)

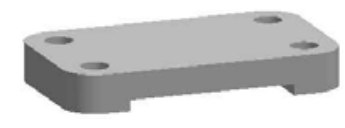

(b)

fig 4. solid of cutting- out 


\section{5 The way to construct the aggregation based on the intersect characteristic}

The way to construct the aggregation based on the intersect characteristic is to get the common part from two bodies, as is shown in the figure 5 .

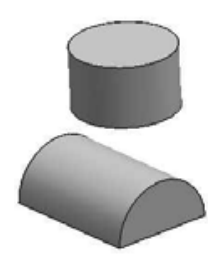

(a)

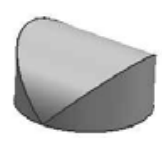

(b)

fig 5. solid of intersect

Every aggregation may be constructed by adding stretching characteristic, revolving characteristic, overlying characteristic, cutting-out characteristic or intersect characteristic lonely, or be constructed by the combination of some characteristics.

\section{THE EXAMPLE OF CONSTRUCTING THE AGGREGATION BASED ON CHARACTERISTIC ADDITION}

Any aggregation constituted of several characteristics is a gathering of characteristics. Figure 6 shows the process of characteristic adding of the bearing pedestal.

The characteristic adding of an aggregation matches expression habit of engineering technician more, which is much close to the machining process of the product, in the same time, strengthens the ability of expressing design ideas and performs creative shape design better.

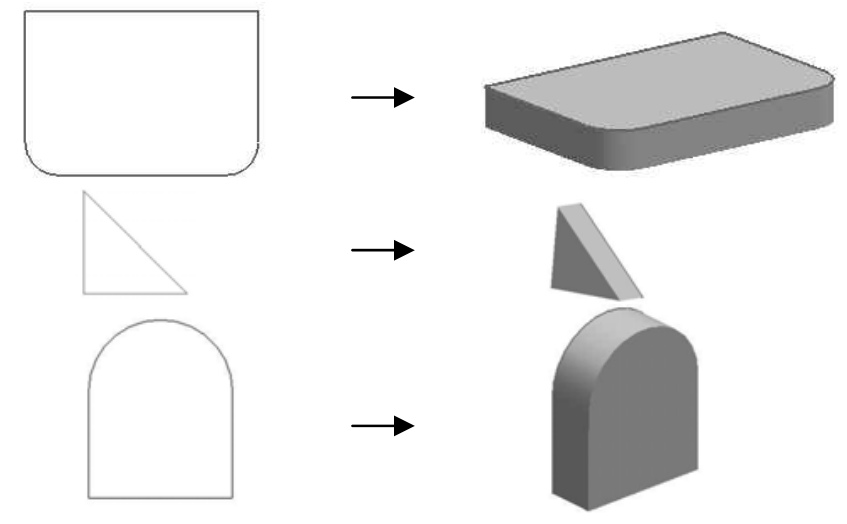

(a) adding stretching characteristic 


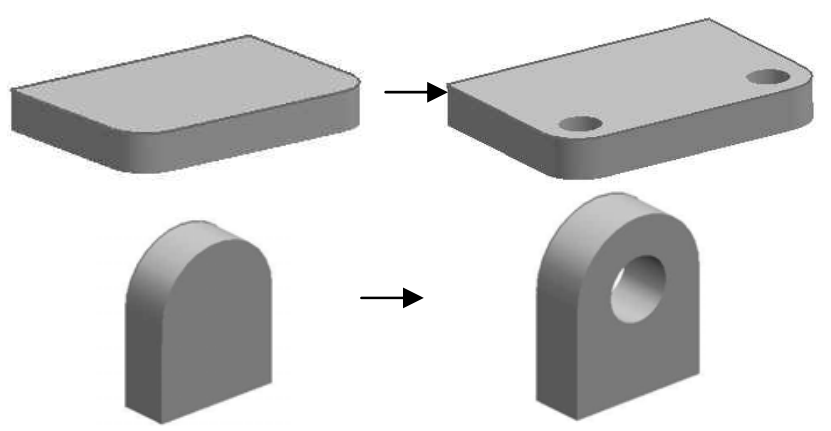

(b) adding cutting-out characteristic

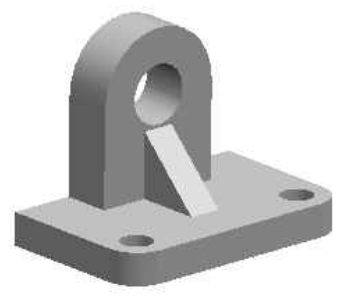

(c) adding overlaying characteristic

Fig 6. the process of characteristic adding of the bearing pedestal.

After configuring an aggregation, you can decompose it into several characteristics again, find out the link between the various characteristics and functionality, the final construction of its three-dimensional models can be remodeled by changing the characteristics of each location and combinations [6]. This union of constitution of the aggregation and engineering practice can foster awareness of modern design and implement the perfect combination of engineering graphics and design.

\section{CONCLUSION}

The graphics educator[7] should in time hold with or go deep into the graphics technical development, integrate the modern engineering design concept, so as to lead new thought and the new technique into graphics course in the appropriate opportunity. The application of Pro/e 3D parameter in the engineering graphics teaching forms new content of course and teaching method, thus, matches exactly the request of a modern era, and therefore will promote the further reform of the engineering graphics [8].

\section{REFERENCES}

[1]. Tong, Bing shu , Tian Lin, Fen Juan, “ The Achievement , Cognition and Problem in The Graphics Educate Innovation in Ten Years” $[\mathrm{J}]$, the thesis collection of the second period engineering graphics academic seminar, pp.43-46.

[2]. Zhan, You gang, “ the china wild couse of the Pro/Engineer” [M], Beijing,China Tsing Hua University Press,2003,pp179.

[3]. Hu, Qing xi , Qing, Peng fei, “ The Research and Application about The Part Model Based on The Characteristic in Manufacture ” [J], mini-micro systems, Vol.20,April, 1999,pp. 32-34. 
[4]. Cheng Yang, Liu, Hai jiang, "The Characteristic modeling in Manufacture” [J], Mechanical and Production Engineering, Vol41, june,2003 pp.15-17.

[5]. Yang, Sheng qiang, “The Model Engineering Graphics” [M], Beijing China Tsing Hua University Press, 2004,pp.101.

[6]. Zhang Haiying, Ma Wei,, Application for Using Pro/E 3D Solid Modeling on Teaching Reform in Engineering Graphic, China educational technology and equipment,2009.,15,pp.117.

[7]. Jiang Hong, Zhang Pei yun, Wu, Ju long, "Enlightenment from American Teaching Materials of Engineering Graphics to Graphics Education Reform and Top-quality Course Construction in China” [J], Journal of Engineering Graphics june,2006,pp56.

[8]. Zhang ,Peiyun, Wu, Ju long, The Enlightenments about Graphics Education Reform and Top-Quality Course Construction Obtained from Teaching Materials of Engineering Graphics of U.S.A, The Journal of Engineering Graphics, june,2006: pp.145-147. 